INPLASY

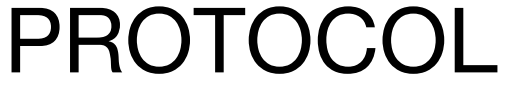

To cite: Zhang et al. The potential diagnostic accuracy of let-7 family for cancer: a meta-analysis. Inplasy protocol 202130013. doi:

10.37766/inplasy2021.3.0013

Received: 06 March 2021

Published: 06 March 2021

Corresponding author:

Shuai-Shuai Gao

631192403@qq.com

Author Affiliation:

Xi'an Daxing Hospital, Shaanxi, China

Support: None.

Review Stage at time of this submission: Data analysis.

Conflicts of interest:

None declared.

\section{The potential diagnostic accuracy of let-7 family for cancer: a meta-analysis}

\author{
Zhang, WT1; Zhang, GX²; Gao, SS³.
}

Review question / Objective: Whether the let-7 family can be used as a diagnostic tool for cancer patients?

Condition being studied: In recent years, more and more studies have verified the possibility of let-7 family as effective non-invasive biomarkers for cancer. Jeong et al. proposed that let-7a can be used as a high-efficiency biomarker for non-small cell lung cancer (NSCLC) with a sensitivity of $90 \%$ and a specificity of $90 \%$. However, Chen et al. found that let- 7 has low diagnostic efficiency for NSCLC with a sensitivity of $67 \%$ and a specificity of $77 \%$. In addition, Lee $\mathbf{C H}$ et al. found that let-7c has a higher diagnostic value for breast cancer (BC), with a sensitivity of $82 \%$ and a specificity of $100 \%$. Whereas Fedorko et al. got a result of $65 \%$ sensitivity and $62 \%$ specificity when let-7c was used for detection of renal cell carcinoma (RCC). The diagnostic efficacy of let-7 family for various cancers is satisfactory but inconsistent. Therefore, we conducted this meta-analysis to comprehensively evaluate the diagnostic accuracy of let-7 family in the diagnosis of cancer.

INPLASY registration number: This protocol was registered with the International Platform of Registered Systematic Review and Meta-Analysis Protocols (INPLASY) on 06 March 2021 and was last updated on 06 March 2021 (registration number INPLASY202130013).

\section{INTRODUCTION}

Review question / Objective: Whether the let-7 family can be used as a diagnostic tool for cancer patients?

Condition being studied: In recent years, more and more studies have verified the possibility of let-7 family as effective noninvasive biomarkers for cancer. Jeong et al. proposed that let-7a can be used as a highefficiency biomarker for non-small cell lung cancer (NSCLC) with a sensitivity of $90 \%$ and a specificity of $90 \%$. However, Chen et al. found that let-7 has low diagnostic efficiency for NSCLC with a sensitivity of 
$67 \%$ and a specificity of $77 \%$. In addition, Lee $\mathrm{CH}$ et al. found that let-7c has a higher diagnostic value for breast cancer (BC), with a sensitivity of $82 \%$ and a specificity of $100 \%$. Whereas Fedorko et al. got a result of $65 \%$ sensitivity and $62 \%$ specificity when let-7c was used for detection of renal cell carcinoma (RCC). The diagnostic efficacy of let-7 family for various cancers is satisfactory but inconsistent. Therefore, we conducted this meta-analysis to comprehensively evaluate the diagnostic accuracy of let-7 family in the diagnosis of cancer.

\section{METHODS}

Search strategy: We conducted a comprehensive search for related articles published up to October 23, 2020 in PubMed, EMBASE, Web of Science, Cochrane Library, Google Scholar, Wanfang Database and China National Knowledge Infrastructure (CNKI) according to the PRISMA statement. Without language restrictions and limited to publications with human subjects, the medical subject headlines (MeSH) terms and keywords were used as follows: "let-7 microRNA" or "miR-let-7" or "let-7" or "hsa-let-7" and "cancer" or "cancers" or "neoplasm" or "neoplasms". In addition, in order to make article retrieval more comprehensive, we manually searched the reference list of related comments to obtain additional articles.

Participant or population: The cancer patient must be diagnosed by histopathological examination and has not received any treatment.

Intervention: Detected the expression level of let-7 family in cancer patients.

Comparator: In the control grupo, the expression level of let-7 family were detected in healthy people o patients with benign tumors.

Study designs to be included: All available estudies that assessed the diagnostic capacity of let-7 family for cancers detection will be included in this metaanalysis.

Eligibility criteria: Investigators screened literatures based on the inclusion criteria: (1) studies aim to evaluated the diagnostic capacity of let-7 family for cancers detection; (2) all cancer patients have been diagnosed through the gold standard test (namely by histopathology examinations); (3) all cancer patients have not received any treatment; (4) healthy people or benign hyperplasia were used as the control; (5) studies contained sufficient data on sensitivity, specificity and sample size to construct a diagnostic two-by-two table. In contrast, the exclusion criteria were: (1) duplicate reports or publications with incomplete information; (2) studies focused on survival or prognosis of cancers; (3) patients who have received treatment (surgery, chemotherapy, radiotherapy); (4) microRNA let-7 obtained from cell lines or animals and (5) comments, reviews, case reports, letters to the editors and systematic reviews or meta-analysis.

Information sources: We conducted a comprehensive search for related articles published up to October 23, 2020 in PubMed, EMBASE, Web of Science, Cochrane Library, Wanfang Database and China National Knowledge Infrastructure (CNKI) according to the PRISMA statement. Without language restrictions and limited to publications with human subjects.

Main outcome(s): The main outcomes included pooled sensitivity, specificity, positive likelihood ratio (PLR), negative likelihood ratio (NLR), diagnostic odds ratio (DOR), and we generated the summary receiver operating characteristics (SROC) curve and calculated the value of area under the curve (AUC).

Data management: The data of the included studies were extracted independently by two investigators, which included the first author's name, publication year, country, let-7 family number, differentiated expression (up or downregulated), cancer types, sample size, specimen source, relevant statistical data 
required and methodological quality information.

Quality assessment / Risk of bias analysis: Two investigators independently assessed the quality of the included studies using the Quality Assessment for Diagnostic Accuracy Studies-2 (QUADAS-2) tool. The publication bias was analyzed using the Deek's funnel plot, in which bias was considered to be significant if $P$-value was less than 0.05. Any disagreements were resolved by a third investigator.

Strategy of data synthesis: All statistical analyses were performed using Review Manager 5.2 and STATA version 13.0. The number of true positives, false positives, false negatives, and true negatives in patients from each study was extracted to estimate the pooled sensitivity, specificity, positive likelihood ratio (PLR), negative likelihood ratio (NLR), diagnostic odds ratio (DOR), and we generated the summary receiver operating characteristics (SROC) curve and calculated the value of area under the curve (AUC). AUC were used to evaluate the diagnostic efficacy: $A U C=$ 1.00 is perfect, AUC $>0.90$ is excellent, AUC $>0.80$ is good, AUC $<0.80$ is medium. The heterogeneity was estimated based on 12 statistic. It indicated significant heterogeneity if $\mathbf{I 2}$ value is greater than $50 \%$, and then a random effects model is performed.

Subgroup analysis: We conducted a subgroup analysis to find probable sources of heterogeneity, which included the country, miRNA profiling, regulation mode, sample size, specimen types, and types of cancer.

Sensitivity analysis: Sensitivity analysis of each parameter was carried out by one-byone elimination method to assess the reliability and robustness of the aggregation results.

Language: Without language restrictions.

Country(ies) involved: China.
Keywords: let-7 family, cancer, diagnosis, meta-analysis.

Dissemination plans: We will disseminate the results of this meta-analysis by publishing the manuscript in a peerreviewed journal or presenting the fingdings at a relevant conference.

Contributions of each author:

Author 1 - Wen-Ting Zhang Conceptualization, Data Curation, Formal analysis, Investigation, Methodology, Software, Supervision, Visualization, Writing-original draft.

Email: 724521882@qq.com

Author 2 - Guo-Xun Zhang Conceptualization, Data Curation, Investigation, Methodology, Visualization, Writing-original draft.

Email: guoxun.zhang@hotmail.com

Author 3 - Shuai-Shuai Gao Conceptualization, Project administration, Resources, Supervision, Visualization, Validation, Writing-review \& editing.

Email: 631192403@qq.com 